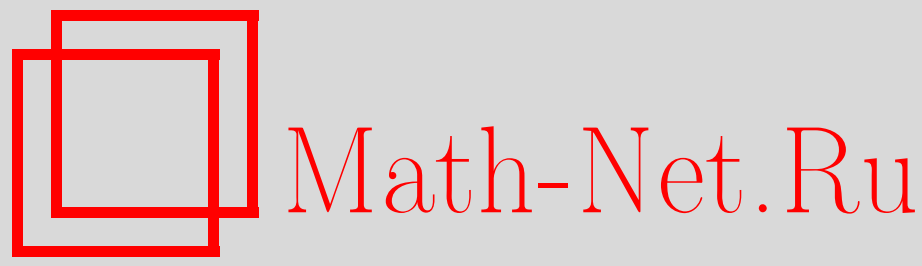

3. 3. Алисултанов, Р. П. Мейланов, Некоторые вопросы теории квантово-статистических систем, обладающих энергетическим спектром дробно-степенного типа, TMФ, 2012, том 173, номер 1, 135-148

DOI: https://doi.org/10.4213/tmf8328

Использование Общероссийского математического портала Math-Net.Ru подразумевает, что вы прочитали и согласны с пользовательским соглашением http://www.mathnet.ru/rus/agreement

Параметры загрузки:

IP : 54.196 .121 .252

26 апреля 2023 г., 14:41:46

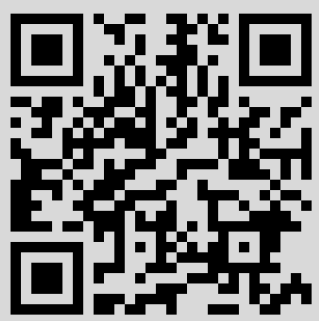




\title{
ФИЗИКА
}

Том 173, № 1

октябрь, 2012

\section{НЕКОТОРЫЕ ВОПРОСЫ ТЕОРИИ КВАНТОВО-СТАТИСТИЧЕСКИХ СИСТЕМ, ОБЛАДАЮЩИХ ЭНЕРГЕТИЧЕСКИМ СПЕКТРОМ ДРОБНО-СТЕПЕННОГО ТИПА}

\begin{abstract}
Рассмотрена задача об эффективном потенциале взаимодействия в квантовой системе многих частиц, приводящем к дробно-степенно́му закону дисперсии. Показано, что переход к производным дробного порядка эквивалентен введению парного межчастичного потенциала. Рассмотрен случай вырожденного электронного газа. С помощью уравнения Ван-дер-Ваальса исследовано уравнение состояния для систем, обладающих дробно-степенным спектром. Получена связь между постоянной Ван-дер-Ваальса и феноменологическим параметром $\alpha$ - порядком дробной производной. Получено соотношение между энергией, давлением и объемом для таких систем; коэффициент перед тепловой энергией есть простая функция от $\alpha$. Рассматривается бозе-эйнштейновская конденсация в системе с дробно-степеннь́м спектром. В рассматриваемом случае критическая температура конденсации для значений $1<\alpha<2$ больше, чем в случае идеальной системы, когда $\alpha=2$.
\end{abstract}

Ключевые слова: дробные производные, неквадратичный спектр, функция Грина, уравнение Ван-дер-Ваальса, бозе-эйнштейновская конденсация.

\section{1. ВВЕДЕНИЕ}

Системы с гамильтонианами, содержащими неквадратичную степень импульса, всегда привлекали внимание исследователей. Это связано прежде всего с тем, что в реальных системах вследствие их неидеальности почти никогда не реализуются квадратичные зависимости энергии от импульса. С другой стороны, систему со сложным законом дисперсии практически не удается точно описать в рамках существующей теории. Поэтому во многих случаях прибегают к приближениям, которые заключаются в представлении неидеальной системы частиц в виде газа свободных квазичастиц с неквадратичной зависимостью энергии от импульса.

* Институт общей физики им. А. М. Прохорова РАН, Москва, Россия. E-mail: zaur0102@gmail.com

${ }^{\dagger}$ Институт проблем геотермии Дагестанского научного центра РАН, Махачкала, Россия. E-mail: lanten50@mail.ru 
Неквадратичная зависимость энергии от импульса приводит к достаточно интересным эффектам. Укажем здесь некоторые. Еще Лифшицем и Кагановым [1] было отмечено, что в случае неквадратичной зависимости энергии электрона от импульса период обращения электронов в магнитном поле, а следовательно, и резонансная частота зависят от приложенного электрического поля. Этот эффект можно использовать для анализа формы изоэнергетических поверхностей в полупроводниках. Интересные результаты были получены в работе [2], где рассматривались высокочастотные электромагнитные явления в полупроводниках с неквадратичным законом дисперсии носителей тока. В работе [3] была рассмотрена электронная хаотичность в одномерной сверхрешетке, обусловленная неквадратичным законом дисперсии. А в работе [4] исследовались нелинейные электромагнитные волны в стохастизированном электронном газе с неквадратичной зависимостью энергии от импульса, и было показано, что отклонение от квадратичной зависимости приводит к замедлению волн.

Другой фактор, усиливающий интерес к системам с неквадратичной зависимостью энергии от импульса, связан с проникновением в физику идей так называемого дробного исчисления, основанного на математическом аппарате интегродифференцирования дробного порядка [5], [6]. Для систем, описываемых дифференциальными уравнениями дробного порядка, характерно отклонение зависимости энергии от импульса от квадратичной и наличие нелокальности дробно-степенно́го характера. Такое отклонение приводит, в частности, к распределениям вероятностей дробно-степенно́го типа. Эти распределения оказываются необычайно устойчивыми [7], [8]. Использование этого аппарата позволяет с большой точностью интерпретировать сложные экспериментальные данные для таких явлений, как аномальная диффузия [6], теплоперенос в средах со сложной структурой [9], дисперсионный перенос в полупроводниках [10], расчет термодинамических свойств поверхностей [11] и т.д. Кроме того, использование этого аппарата позволило предсказать ряд новых эффектов. Так, например, в работах [12], [13] было показано, что в рамках концепции фрактонов удается добиться сравнительно больших температур перехода в сверхпроводящее состояние, что делает интересным аппарат дробного интегродифференцирования в теории сверхпроводимости. Следует отметить, что нелокальные взаимодействия степенно́го типа рассматривались еще в работах Дайсона в применении к фазовым переходам в ферромагнетиках [14]-[16]. В работе [17] рассматривались нелокальные взаимодействия такого типа в кристаллической решетке и были получены дробные кинетические уравнения. Особенности дробной квантовой механики рассматривались в работах [18]--[21], где было проведено исследование систем с гамильтонианами, содержащими нецелую степень импульса. Необходимо отметить, что заметный вклад в обобщение уравнений на случай фрактальных сред, а также в исследование систем, описываемых уравнениями в дробных операторах, внес Тарасов. Так, например, в работах [22]-[24] была рассмотрена дробная статистическая механика, получены дробные аналоги уравнений Лиувилля и Боголюбова, а также кинетические уравнения с дробными производными.

Наконец, необходимо отметить, что полученная недавно двумерная модификация атомов углерода - графен [25], [26] - является системой с гамильтонианом, содержащим неквадратичную степень импульса. Действительно, электроны графена вблизи так называемой точки Дирака обладают линейным законом дисперсии [27]. 
Исследование графена очень важно для современной науки, так как благодаря своим уникальным свойствам он может стать очень перспективным материалом для нанотехнологий.

Идея дробно-степенно́й зависимости энергии от импульса лежит также в основе работы [28], где подробно был рассмотрен вопрос о возможном способе введения и физической интерпретации дробного интегродифференцирования в квантовой статистической физике. Эта идея развивается также и в настоящей работе. Напомним основную суть развиваемой теории. Исходным пунктом исследований является уравнение для функции Грина, записанное в дробных производных по пространственным координатам в виде

$$
\left[i \hbar \frac{\partial}{\partial t_{1}}+E_{0} \frac{\hbar^{\alpha}}{p_{0}^{\alpha}} \Delta_{1}^{\alpha / 2}\right] G\left(1,1^{\prime}\right)=\delta\left(1-1^{\prime}\right),
$$

где 1 означает $r_{1} t_{1}, 1^{\prime}-r_{1^{\prime}} t_{1^{\prime}}, E_{0}$ и $p_{0}$ - характеристические энергия и импульс системы, $\Delta_{1}^{\alpha / 2}$ - дробная производная Рисса, определенная в одномерном случае каK [29]

$$
\Delta_{x}^{\alpha / 2} f(x)=\frac{1}{\Gamma(2-\alpha) \cos ((\pi / 2)(2-\alpha))} \frac{\partial^{2}}{\partial x^{2}} \int_{-\infty}^{\infty} \frac{f(\xi) d \xi}{|x-\xi|^{\alpha-1}},
$$

где $1<\alpha \leqslant 2$. Возможность нестрогого ограничения сверху значений, принимаемых параметром $\alpha$, была доказана в работе [30]. Спектр системы, описываемой уравнением (1), как легко показать, имеет вид

$$
\omega(p)=\frac{E_{0}}{p_{0}^{\alpha}}|p|^{\alpha}+\text { const } .
$$

В настоящей работе продолжается начатое в работе [28] исследование статистических свойств систем, обладающих энергетическим спектром дробно-степенно́го типа (2). На наш взгляд, настоящая работа интересна по нескольким причинам. Во-первых, в работе обсуждаются физические условия, соответствующие возникновению в уравнении для функции Грина дробного оператора Рисса. Это позволит сделать определенные выводы исследователям, использующим уравнения в таких операторах в некоторой степени формально. Во-вторых, рассмотрены некоторые важные свойства систем с энергетическим спектром дробно-степенно́го типа. Последнее дает важную информацию о связи феноменологического параметра - порядка дробной производной - с естественными параметрами реальной статистической системы (межчастичный потенциал взаимодействия, постоянная Ван-дер-Ваальса, затухание возбужденных состояний и т.п.).

\section{2. ЭФФЕКТИВНЫЙ ПОТЕНЦИАЛ, ПРИВОДЯЩИЙ К ДРОБНЫМ ПРОИЗВОДНЫМ В УРАВНЕНИИ ДЛЯ ФУНКЦИИ ГРИНА}

Обсудим возможность появления в уравнении для функции Грина дробного оператора Рисса. Попытаемся определить явный вид межчастичного потенциала взаимодействия в многочастичной системе, приводящего к такому оператору в основном уравнении. Общее уравнение для функции Грина при наличии взаимодействия между частицами записывается как [31]

$$
\left(i \hbar \frac{\partial}{\partial t_{1}}+E_{0} \frac{\hbar^{2}}{p_{0}^{2}} \Delta_{1}\right) G\left(1 ; 1^{\prime}\right)-\int_{0}^{-i \beta} d \bar{t}_{1} d \bar{r}_{1} \Sigma\left(r_{1} t_{1} ; \bar{r}_{1} \bar{t}_{1}\right) G\left(\bar{r}_{1} \bar{t}_{1} ; r_{1^{\prime}} t_{1^{\prime}}\right)=\delta\left(1-1^{\prime}\right),
$$


где $\Sigma\left(1 ; 1^{\prime}\right)$ есть массовый оператор, содержащий всю информацию о взаимодействии частиц между собой. Применяя фурье-преобразование к обеим частям уравнения (3), получим

$$
\left[\omega-\frac{E_{0}}{p_{0}^{2}} p^{2}-\Sigma(p, \omega)\right] G(p, \omega)=1 .
$$

Согласно уравнению (4) спектр системы определяется как $\omega(p)=\left(E_{0} / p_{0}^{2}\right) p^{2}+$ $\operatorname{Re} \Sigma(p, \omega)$. Спектру (2), как легко видеть, соответствует массовый оператор $\Sigma(p)=$ $E_{0}\left(p_{0}^{-\alpha}|p|^{\alpha}-p_{0}^{-2} p^{2}\right)$. С другой стороны, массовый оператор в общем случае определяется выражением [31]

$$
\Sigma(p, \omega) G(p, \omega)=i \int d r d t e^{-i \omega t / \hbar+i p r / \hbar} \int d r_{2} V\left(r_{1}-r_{2}\right) G_{2}\left(12,1^{\prime} 2^{\prime}\right)_{t_{1}=t_{2}},
$$

или

$$
\frac{E_{0}\left(p_{0}^{-\alpha}|p|^{\alpha}-p_{0}^{-2} p^{2}\right)}{\omega-E_{0} p_{0}^{-\alpha}|p|^{\alpha}}=i \int d r d t e^{-i \omega t / \hbar+i p r / \hbar} \int d r_{2} V\left(r_{1}-r_{2}\right) G_{2}\left(12,1^{\prime} 2^{\prime}\right) t_{1}=t_{2},
$$

где $G_{2}\left(12,1^{\prime} 2^{\prime}\right)$ - двухчастичная функция Грина, $V(r)$ - межчастичный потенциал взаимодействия. Выражение (6) и определяет те условия, т. е. тот потенциал $V(r)$, который приводит к спектру (2). Однако решить это точное уравнение в общем случае нельзя. Воспользуемся аппроксимацией для массового оператора. Можно показать, что массовый оператор для данной системы не содержит столкновений частиц, поэтому ограничимся аппроксимациями низшего порядка. В аппроксимации Хартри-Фока для фермионного газа имеем

$$
\Sigma_{\mathrm{HF}}(p)=n \int V(r) d r-\int \frac{d p^{\prime}}{(2 \pi)^{3}} \frac{V\left(p-p^{\prime}\right)}{e^{\beta\left[E_{0} p_{0}^{-\alpha}\left|p^{\prime}\right|^{\alpha}-\mu\right]}+1},
$$

где $\beta=1 /(k T), \mu$ - химический потенциал, $n$ - концентрация частиц в трансляционно-инвариантной системе. Очевидно, что видоизменить зависимость энергии от импульса можно только за счет фоковской части массового оператора. С учетом этого запишем

$$
E_{0}\left(p_{0}^{-\alpha}|p|^{\alpha}-p_{0}^{-2} p^{2}\right)=-\int \frac{d^{3} p^{\prime}}{(2 \pi \hbar)^{3}} \frac{V\left(p-p^{\prime}\right)}{e^{\beta\left[E_{0} p_{0}^{-\alpha}\left|p^{\prime}\right|^{\alpha}-\mu\right]}+1} .
$$

Спектр такой системы имеет вид $\omega(p)=E_{0} p_{0}^{-\alpha}|p|^{\alpha}+n \int V(r) d r$, и ему соответствует уравнение для функции Грина

$$
\left[\omega-E_{0} p_{0}^{-\alpha}|p|^{\alpha}-n \int V(r) d r\right] G\left(1,1^{\prime}\right)=\delta\left(1-1^{\prime}\right),
$$

которое после фурье-преобразования приводит к уравнению

$$
\left[i \hbar \frac{\partial}{\partial t_{1}}+E_{0} \frac{\hbar^{\alpha}}{p_{0}^{\alpha}} \Delta_{1}^{\alpha / 2}+\int V\left(r_{1}-r_{2}\right)\left\langle n\left(r_{2}\right)\right\rangle d r_{2}\right] G\left(1,1^{\prime}\right)=\delta\left(1-1^{\prime}\right) .
$$

Заметим, что это уравнение отличается от уравнения (1) наличием хартриевского члена. В работе [28] мы пренебрегали хартриевским членом в уравнении для функции Грина. В настоящей работе мы также последуем этому приближению. 
Займемся непосредственно выяснением условий, приводящих к спектру (2). Для этого необходимо решить интегральное уравнение (7) относительно потенциала $V$. Применяя к обеим частям уравнения (7) фурье-преобразование, получим

$$
V(r)=E_{0} \int \frac{d^{D} k}{(2 \pi)^{D}}\left(k_{0}^{-2} k^{2}-k_{0}^{-\alpha}|k|^{\alpha}\right) e^{-i k r} \cdot\left[\int \frac{d^{D} k}{(2 \pi)^{D}} \frac{e^{-i k r}}{e^{\beta\left[E_{0} k_{0}^{-\alpha}|k|^{\alpha}-\mu\right]}+1}\right]^{-1},
$$

где мы перешли к переменным $k=p / \hbar, V(r)=\int\left(d^{D} k /(2 \pi)^{D}\right) V(k) e^{-i k r}, D=1,2,3-$ размерность рассматриваемой системы. Чтобы определить $\int|p|^{\alpha} e^{-i p \xi} p^{D-1} d p$, воспользуемся обычным приемом:

$$
\begin{aligned}
\int|p|^{\alpha} e^{-i p \xi} p^{D-1} d p & =\int_{0}^{\infty} p^{\alpha+D-1}\left(e^{i p \xi}+e^{-i p \xi}\right) d p= \\
& =\lim _{\delta \rightarrow 0} \int_{0}^{\infty} p^{\alpha+D-1}\left(e^{i p(\xi+i \delta)}+e^{-i p(\xi-i \delta)}\right) d p .
\end{aligned}
$$

Тогда получим

$$
V(r)=E_{0}\left(k_{0}^{-2} \frac{\Gamma(2+D)}{r^{2+D}}-k_{0}^{-\alpha} \frac{\Gamma(\alpha+D)}{r^{\alpha+D}}\right) \cdot\left[\int \frac{d^{D} k}{(2 \pi)^{D}} \frac{e^{-i k r}}{e^{\beta\left[E_{0} k_{0}^{-\alpha}|k|^{\alpha}-\mu\right]}+1}\right]^{-1},
$$

Интеграл $K(r)$ не вычисляется в общем виде. Поэтому рассмотрим случай малой плотности, когда $e^{-\beta \mu} \gg 1$. Тогда

$$
K(r)=e^{\beta \mu} \int \frac{d^{D} k}{(2 \pi)^{D}} e^{-\beta E_{0} k_{0}^{-\alpha}|k|^{\alpha}} e^{-i k r} .
$$

Этот интеграл положим равным $K(r)=A e^{\beta \mu} e^{-|r|^{\alpha} / \beta E_{0} k_{0}^{-\alpha}}$, где $A$ - константа, появляющаяся при интегрировании. Таким образом, для потенциала взаимодействия (8) получаем

$$
V(r)=\frac{E_{0}}{A} e^{-\beta \mu} e^{|r|^{\alpha} / \beta E_{0} k_{0}^{-\alpha}}\left(k_{0}^{-2} \frac{\Gamma(2+D)}{r^{2+D}}-k_{0}^{-\alpha} \frac{\Gamma(\alpha+D)}{r^{\alpha+D}}\right) .
$$

Мы получили тот потенциал, который в данном подходе приводит к дробной производной Рисса по координате. При $\alpha=2$ этот потенциал равен нулю. Пусть рассматриваемая система находится при температуре $T \sim 10^{\circ} \mathrm{K}$, и масса частиц имеет порядок массы протона, т. е. $M \approx 10^{-28}$ кг. Характеристическую энергию возьмем равной $E_{0}=0.1$ эВ, а для характерного волнового числа по формуле $E_{0}=k_{0}^{2} \hbar^{2} / 2 M$ получаем $k_{0} \approx 10^{10} \mathrm{M}^{-1}$. Кроме того, предположим, что $e^{-\beta \mu} \approx 20$. Тогда полученный потенциал в случае трехмерной системы ведет себя так, как показано на рис. 1. Энергия дается в единицах $E_{0} / A$.

По своему физическому смыслу производная дробного порядка по координате означает учет пространственных корреляций. Как видно из рис. 1, переход к производным дробного порядка действительно эквивалентен введению парного межчастичного потенциала, приводящего к неидеальности газа. Однако в отличие от традиционного межчастичного потенциала, который имеет чисто потенциальную природу и не зависит от импульса, в данном случае мы имеем принципиально иной 


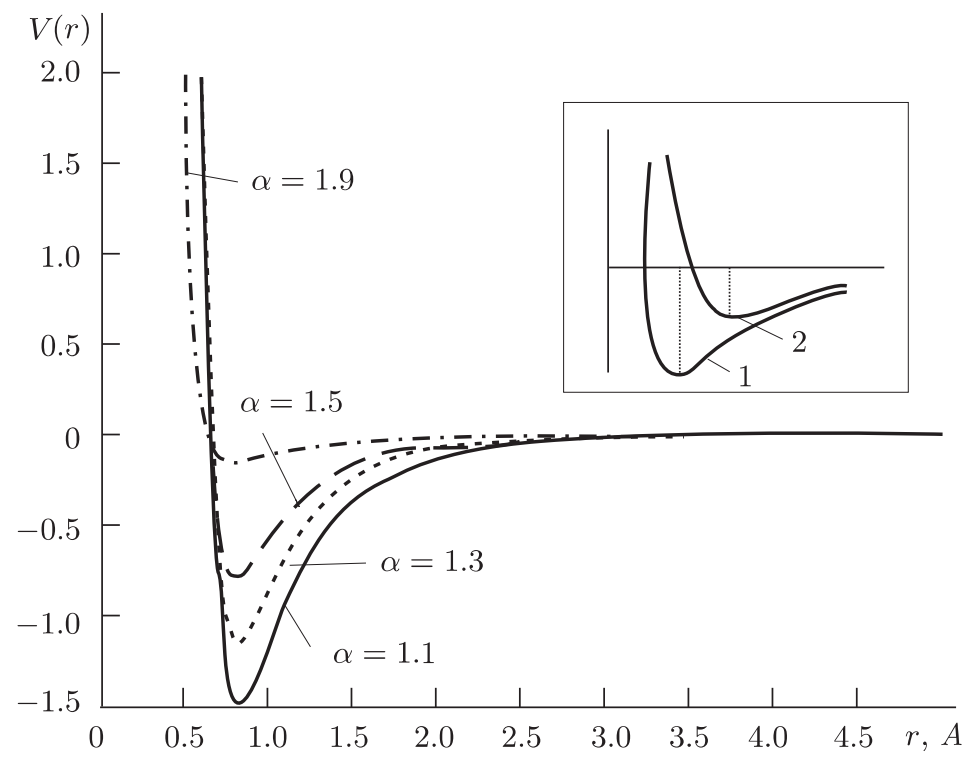

Рис. 1. Потенциал взаимодействия (9) при различных значениях $\alpha$. Для сравнения на врезке приведен потенциал межатомного взаимодействия жидкого гелия (1 - потенциальная энергия, 2 - энергия) (см., например, работу [32]).

случай. Дело в том, что переход к дробной производной, приводящий к дробному спектру, означает учет эффективного взаимодействия между частицами, причем чрезвычайно важно, что это взаимодействие фактически неявно зависит от импульса. В этой связи отметим, что рассмотрение случая, когда энергия взаимодействия между частицами зависит от импульса в традиционной статистической физике, в принципе невозможно. Таким образом, переход к производным дробного порядка по координате фактически означает учет зависимости энергии взаимодействия от импульса и тем самым расширяет границы применимости статистической физики, позволяя также учитывать зависимость энергии взаимодействия от импульсов.

Проанализируем полученный потенциал на примере системы заряженных частиц. В такой системе потенциал взаимодействия между частицами в общем случае определяется согласно выражению [32]

$$
V(r)=\int \frac{e^{2} e^{-i k r} d k}{k^{2} \varepsilon(k)} .
$$

Величина $e^{2} / k^{2}$ есть фурье-образ кулоновского взаимодействия в трехмерной системе, $\varepsilon(k)$ - диэлектрическая проницаемость в среде с пространственной дисперсией. В среде без дисперсии имеем $\varepsilon=$ const, а следовательно, $V(r)=q^{2} /(\varepsilon r)$, т. е. приходим к закону Кулона. Если диэлектрическая проницаемость имеет вид $\varepsilon(k)=1+k_{0}^{2} / k^{2}$, то приходим к дебаевской экранировке $V(r)=q^{2} e^{-k_{0} r} / r$, где $k_{0}^{-1}$ 

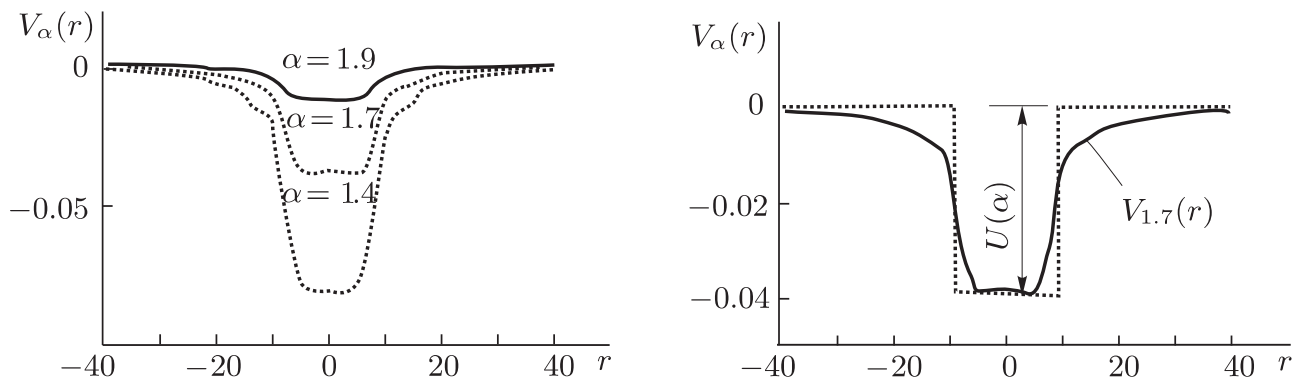

Рис. 2. Межчастичный потенциал взаимодействия в вырожденном электронном газе, приводящий к энергетическому спектру дробно-степенно́го типа (2).

есть радиус Дебая. Представляет интерес решение уравнения

$$
V_{\alpha}(r)=\int \frac{q^{2} e^{-i k r} d k}{k^{2} \varepsilon_{\alpha}(k)},
$$

где $V_{\alpha}(r)$ есть выражение $(9)$, относительно $\varepsilon_{\alpha}(k)$. В этом случае можно утверждать, что в системе заряженных частиц с диэлектрической проницаемостью $\varepsilon_{\alpha}(k)$ межчастичный потенциал определяется выражением (9), что приводит к дробно-степенно́му спектру и, следовательно, к дробной производной Рисса в уравнении для функции Грина.

\section{3. ВЫРОЖДЕННЫЙ ЭЛЕКТРОННЫЙ ГАЗ}

Рассмотрим случай вырожденного электронного газа. В этом случае необходимо положить $\left[e^{\beta\left[E_{0} p_{0}^{-\alpha}\left|p^{\prime}\right|^{\alpha}-\mu\right]}+1\right]^{-1}=\Theta\left(E_{\mathrm{F}}-E_{0} p_{0}^{-\alpha}|p|^{\alpha}\right)$. Кроме того, положим $E_{0}=E_{\mathrm{F}}$ и $p_{0}=p_{\mathrm{F}}$. Учитывая это, из выражения (8) получим

$$
V_{\alpha}(r)=E_{\mathrm{F}} \operatorname{Re}\left\{\frac{\int_{0}^{k_{\mathrm{F}}} k^{2} d k\left(k_{\mathrm{F}}^{-2} k^{2}-k_{\mathrm{F}}^{-\alpha} k^{\alpha}\right) e^{-i k r}}{\int_{0}^{k_{\mathrm{F}}} k^{2} d k e^{-i k r}}\right\} .
$$

На рис. 2 изображена зависимость межчастичного потенциала (10) от расстояния между частицами. Энергия дается в единицах энергии Ферми, а расстояние - в ангстремах. Потенциал взаимодействия, как легко видеть из рис. 2, представляет собой некоторую потенциальную яму, глубина которой зависит от параметра $\alpha$.

Другой важный вывод состоит в том, что взаимодействие в данном случае носит характер притяжения, и поэтому можно сказать, что мы имеем дело с явлением, аналогичным явлению сверхпроводимости, при котором электроны за счет обмена фононами начинают притягиваться и образовывать куперовские пары. Кроме того, в данном случае интенсивность электрон-электронного притяжения есть функция от параметра $\alpha$. По-видимому, в такой системе должен реализовываться следующий эффект. При определенной температуре в рассматриваемой системе электроны локализуются в потенциальной яме, приведенной на рис. 2. Переход в такое состояние назовем f-переходом. Температура f-перехода определяется, очевидно, глубиной 
ямы, которая в свою очередь зависит от параметра $\alpha$. Таким образом, для температуры перехода имеем $U(\alpha)=k_{\mathrm{B}} T_{\mathrm{f}}$. Сделаем некоторые оценки. Для значения $\alpha=1.9$ глубина ямы порядка $U(\alpha=1.9)=0.01$ эВ. Отсюда находим, что для этого случая $T_{\mathrm{f}} \approx 100 \mathrm{~K}$. Аналогично для значения $\alpha=1.7$, например, получим $T_{\mathrm{f}} \approx 400 \mathrm{~K}$.

\section{4. ИССЛЕДОВАНИЕ УРАВНЕНИЯ СОСТОЯНИЯ ДЛЯ СИСТЕМЫ, ОБЛАДАЮЩЕЙ ДРОБНО-СТЕПЕННЫंМ СПЕКТРОМ}

Рассмотрим вопрос об уравнении состояния газа (для определенности возьмем ферми-газ), обладающего спектром (2) и соответственно описываемого уравнением (1). Распределение Ферми для такого газа имеет вид $n_{\alpha}(\beta, p)=\left(e^{\beta\left(|p|^{\alpha}-\mu\right)}+1\right)^{-1}$, где используется система единиц, в которой несущественные на данном этапе постоянные равны единице, т. е. $E_{0}=1, p_{0}=1$. Уравнение состояния для системы с таким распределением получено в работе [28], где использовалось соотношение $d P=n d \mu, P$ - давление. Для нахождения дифференциала от химического потенциала было продифференцировано $n_{\alpha}(\beta, p)$. Основное соображение, использованное в работе [28] при совершении таких действий над функцией $n_{\alpha}(\beta, p)$, заключается в следующем. Параметр $\alpha$ неявно содержит в себе эффективное взаимодействие между частицами. Это взаимодействие, естественно, определяется концентрацией частиц. Следовательно, при дифференцировании следует иметь в виду, что параметр $\alpha$ не является постоянной величиной, а есть функционал плотности. Это значит, что по этому параметру также необходимо провести дифференцирование. В некотором смысле эти соображения очень похожи на подобные утверждения теории ферми-жидкости. Действительно, в последнем случае распределение записывается как $n(\beta, p)=\left(e^{\beta(\varepsilon(p)-\mu)}+1\right)^{-1}$, где $\varepsilon(p)$ есть энергия квазичастиц, которая в свою очередь является функционалом от плотности, т. е. $\varepsilon=\varepsilon(n, p)$. $\mathrm{C}$ помощью таких соображений в работе [28] было получено уравнение состояния для ферми-системы, обладающей энергетическим спектром дробно-степенного типа. Оно имеет вид

$$
P=\frac{1}{\beta} n+2 \frac{1}{\beta} e^{\beta \mu} \int \frac{d^{3} p}{(2 \pi)^{3}}\left(1-e^{-\beta\left(p^{2}-|p|^{\alpha}\right)}\right) e^{-\beta|p|^{\alpha}},
$$

где $n$ - концентрация идеального газа (при $\alpha=2$ ). Не представляет труда с помощью таких же соображений получить и уравнение состояния для бозе-газа. Из уравнения (11) видно, что степенно́й закон дисперсии приводит в конечном итоге к появлению некоторого добавочного давления в уравнении состояния, т. е. фактически учитывает взаимодействие между частицами. Функция распределения, определяющая это добавочное давление, в сущности представляет собой распределение Вейбулла (растянутая экспонента), которое тесно связано с фрактальными свойствами системы [33]. Это добавочное давление равно нулю при $\alpha=2$, и мы приходим к уравнению состояния идеального газа.

Исследуем уравнение (11). Как было сказано, оно описывает газ взаимодействующих фермионов. Попытаемся сделать некоторые общие замечания относительно 
характера этого взаимодействия с помощью более привычных подходов. Уравнение (11), записанное в виде

$$
P-I_{\alpha}=\frac{N}{V} k T,
$$

где $I_{\alpha}=2 \beta^{-1} e^{\beta \mu} \int\left(d^{3} p /(2 \pi)^{3}\right)\left(1-e^{-\beta\left(p^{2}-|p|^{\alpha}\right)}\right) e^{-\beta|p|^{\alpha}}$, очень похоже на уравнение Ван-дер-Ваальса, которое, однако, не содержит информации о конечности размеров частиц. Это связано с тем, что рассмотренная аппроксимация не учитывает столкновений и того факта, что частицы не могут проникать друг в друга. Если пренебречь эффектами конечности размеров частиц, то уравнение Ван-дер-Ваальса может быть записано как

$$
P-n^{2} a=\frac{N}{V} k T .
$$

Сравнивая его с уравнением (19), получим

$$
n^{2} a=2 \frac{1}{\beta} e^{\beta \mu} \int \frac{d^{3} p}{(2 \pi)^{3}}\left(1-e^{-\beta\left(p^{2}-|p|^{\alpha}\right)}\right) e^{-\beta|p|^{\alpha}} .
$$

С целью получения выражения для химического потенциала прибегнем к приближению, а именно будем использовать выражение для химического потенциала больцмановского идеального газа. Это приближение во многих случаях оправдано [34]. Химический потенциал больцмановского газа с точностью до несущественных постоянных величин есть величина, равная $\mu=\beta^{-1} \ln \left(n \beta^{3 / 2}\right)$ [34]. Тогда имеем

$$
n a=2 \beta^{1 / 2} \int \frac{d^{3} p}{(2 \pi)^{3}}\left(1-e^{-\beta\left(p^{2}-|p|^{\alpha}\right)}\right) e^{-\beta|p|^{\alpha}} .
$$

Для конкретных вычислений необходимо перейти к размерным величинам. В размерных переменных интеграл $I_{\alpha}$ записывается как

$$
I_{\alpha}=2 \frac{1}{\beta} e^{\beta \mu} \int \frac{d^{3} k}{(2 \pi)^{3}}\left(1-e^{-\beta E_{0}\left(k_{0}^{-2} k^{2}-k_{0}^{-\alpha}|k|^{\alpha}\right)}\right) e^{-\beta E_{0} k_{0}^{-\alpha}|k|^{\alpha}},
$$

где, как и выше, $k=p / \hbar$. Химический потенциал больцмановского газа в размерных переменных имеет вид $\mu=\beta^{-1} \ln \left[(n / 2)\left(2 \pi \hbar^{2} m^{-1} \beta\right)^{3 / 2}\right]$ [34]. Для постоянной Ван-дер-Ваальса, следовательно, получим следующее выражение, связывающее эту постоянную с параметром $\alpha$ :

$$
a=\frac{\beta^{1 / 2}}{n}\left(\frac{2 \pi \hbar^{2}}{m}\right)^{3 / 2} \int \frac{d^{3} k}{(2 \pi)^{3}}\left(1-e^{-\beta E_{0}\left(k_{0}^{-2} k^{2}-k_{0}^{-\alpha}|k|^{\alpha}\right)}\right) e^{-\beta E_{0} k_{0}^{-\alpha}|k|^{\alpha}} .
$$

Это соотношение очень важно с точки зрения возможности описания реальных систем методом дробного интегродифференцирования, которое осуществляется с помощью уравнения (1). Эта зависимость приведена на рис. 3. Каждому значению постоянной Ван-дер-Ваальса $a$ ставится в соответствие одно значение параметра $\alpha$, т. е. системы с различными $a$ можно описать уравнениями состояния (12) с параметрами $\alpha$, соответствующими этим $a$. 


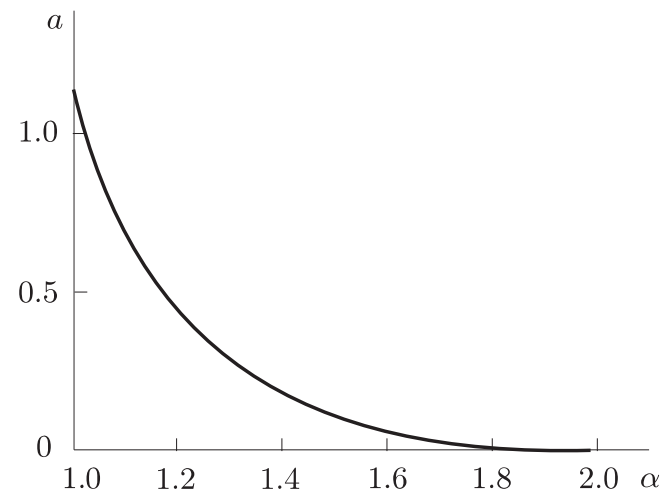

a

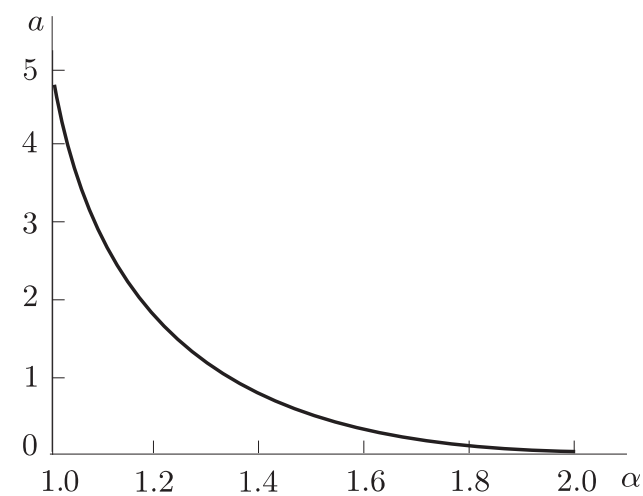

б

Рис. 3. Зависимость постоянной Ван-дер-Ваальса $a$ от параметра $\alpha$ : a $q_{0}^{2} /\left(2 m \beta^{-1}\right)=1 ; б-q_{0}^{2} /\left(2 m \beta^{-1}\right)=2$. При построении кривых условно принято, что $\beta^{-1} / n(\pi)^{3 / 2}=1$.

\section{5. СООТНОШЕНИЕ МЕЖДУ ЭНЕРГИЕЙ, ДАВЛЕНИЕМ И ОБЪЕМОМ}

Представляет интерес получение соотношения между энергией, давлением и объемом для системы с дробно-степеннь́м спектром. В дальнейшем нас будут интересовать лишь качественные зависимости определяемых величин, поэтому мы пренебрежем несущественными постоянными множителями в последующих выражениях. Там, где возникнет необходимость оставить постоянные члены, это будет специально оговорено.

В работе [28] было показано, что для системы с дробно-степенны́м спектром плотность состояний имеет вид $\rho(\omega)=\alpha^{-1} \omega^{D / \alpha-1}$, где $D=1,2,3$ - размерность фазового пространства. Параметр $\alpha$ является существенным, поэтому он будет сохранен во всех выражениях. Кроме того, заметим, что выражение для плотности состояний было получено в работе [28] в системе единиц, в которой $E_{0}=1, p_{0}=1$. Однако, как можно легко понять из введения, эти постоянные входят в выражение для спектра вместе с важным параметром $\alpha$. Приняв условие $E_{0}=1, p_{0}=1$, мы тем самым могли потерять и параметр $\alpha$. Однако легко показать, что даже в самом общем случае, когда сохранены все постоянные, этот параметр входит в соотношение именно таким образом, как это получено в работе [28]. В этом можно легко убедиться путем вывода этого выражения по правилам, использованным в работе [28]. Рассматривая трехмерную систему, имеем $\rho(\omega)=\alpha^{-1} \omega^{3 / \alpha-1}$. Число частиц с энергией в промежутке от $\omega$ до $\omega+d \omega$ при этом есть величина

$$
d N=\frac{V \omega^{3 / \alpha-1} d \omega}{\alpha e^{\beta(\omega-\mu)}+1} .
$$

Величины $\beta$ и $\mu$ определяют термодинамическое состояние системы и поэтому не могут быть опущены. В последнем выражении мы опустили также и спиновую постоянную. Заметим, что все приводимые в этом пункте формулы имеют совершенно аналогичный вид для обеих статистик Ферми и Бозе, отличаясь лишь 
знаком в знаменателе выражения (14). Так, для бозе-газа получим выражение $d N=V \alpha^{-1}\left(e^{\beta(\omega-\mu)}-1\right)^{-1} \omega^{3 / \alpha-1} d \omega$. Интегрируя выражение (14) по энергии, получим полное число частиц в газе:

$$
N=\frac{V}{\alpha} \int_{0}^{\infty} \frac{\omega^{3 / \alpha-1} d \omega}{e^{\beta(\omega-\mu)}+1} .
$$

Вводя переменную интегрирования $z=\beta \omega$, имеем

$$
\frac{N}{V}=\frac{\beta^{-3 / \alpha}}{\alpha} \int_{0}^{\infty} \frac{z^{3 / \alpha-1} d z}{e^{z-\beta \mu}+1} .
$$

Эта формула определяет химический потенциал $\mu$ как функцию от температуры $\beta^{-1}$ и плотности $N / V$. Для большого термодинамического потенциала имеем

$$
\Omega=-\frac{V \beta^{-1}}{\alpha} \int_{0}^{\infty} \omega^{3 / \alpha-1} \ln \left(1+e^{\beta(\mu-\omega)}\right) d \omega .
$$

Интегрируя по частям, получим

$$
\Omega=-\frac{\alpha}{3} \frac{V}{\alpha} \int_{0}^{\infty} \frac{\omega^{3 / \alpha} d \omega}{e^{\beta(\omega-\mu)}+1} .
$$

Найдем с помощью выражения (14) полную энергию системы:

$$
E=\int \omega d N=\frac{V}{\alpha} \int_{0}^{\infty} \frac{\omega^{3 / \alpha} d \omega}{e^{\beta(\omega-\mu)}+1} .
$$

Выражение (16) с точностью до множителя $-\alpha / 3$ совпадает с выражением (17). Имея в виду также, что $\Omega=-P V$, получим выражение

$$
P V=\frac{\alpha}{3} E
$$

Интересно отметить, что значение коэффициента при тепловой энергии определяется степенью зависимости энергии от импульса в законе дисперсии. При $\alpha=2$ получаем соотношение для состояния идеального газа.

\section{6. БОЗЕ-ЭЙНШТЕЙНОВСКАЯ КОНДЕНСАЦИЯ В СИСТЕМЕ С ЭНЕРГЕТИЧЕСКИМ СПЕКТРОМ ДРОБНО-СТЕПЕННО́ГО ТИПА}

Одним из важных вопросов статистической физики является вопрос о переходе системы в бозе-эйнштейновский конденсат. Известно, что неидеальность системы приводит к тому, что температура перехода понижается. При низких температурах в системе становятся существенными эффекты нелокальности, так как при этом взаимодействие между частицами начинает преобладать над энергией теплового движения. В связи с этим представляет интерес рассмотрение явления бозе-эйнштейновского конденсата в системе с нелокальностью дробно-степенно́го характера, т.е. в системе с энергетическим спектром дробно-степенно́го типа (2). Такая нелокальность, как оказалось, приводит к особым результатам и, на наш

6 Теоретическая и математическая физика, т. 173, № 1, 2012 г. 
взгляд, интересна для теории сверхпроводимости. В настоящей работе получена связь критической температуры конденсации бозе-газа с параметром $\alpha$ в законе дисперсии (2).

Для большого термодинамического потенциала трехмерного газа с законом дисперсии (2) имеем

$$
\Omega=\frac{2}{\beta \Gamma(3 / 2)} \frac{V \pi^{3 / 2}}{(2 \pi)^{3}} \int \ln \left[1-e^{-\beta E_{0} k_{0}^{-\alpha}|k|^{\alpha}} e^{\beta \mu}\right] k^{2} d k .
$$

Соответственно для плотности частиц получим

$$
\rho=-\frac{1}{V} \frac{\partial \Omega}{\partial \mu}=\frac{2}{\Gamma(3 / 2)} \frac{\pi^{3 / 2}}{(2 \pi)^{3}} \int \frac{e^{-\beta E_{0} k_{0}^{-\alpha} k^{\alpha}} e^{\beta \mu}}{1-e^{-\beta E_{0} k_{0}^{-\alpha} k^{\alpha}} e^{\beta \mu}} k^{2} d k .
$$

Введем обозначения

$$
\begin{gathered}
\gamma=e^{\beta \mu}, \quad x^{2}=\beta E_{0} k_{0}^{-\alpha} k^{\alpha}, \\
C(\alpha)=\frac{2}{\alpha \Gamma(3 / 2)} \frac{\pi^{3 / 2}}{(2 \pi)^{3}} \frac{1}{E_{0}^{3 / \alpha} k_{0}^{-3}} .
\end{gathered}
$$

Тогда выражение (19) можно переписать в виде

$$
\rho=C(\alpha)\left(\frac{1}{\beta}\right)^{3 / \alpha} \int \frac{e^{-x^{2}} \gamma}{1-e^{-x^{2}} \gamma} x^{6 / \alpha-1} d x
$$

и, далее,

$$
\begin{aligned}
\rho & =C(\alpha)\left(\frac{1}{\beta}\right)^{3 / \alpha}\left[\int x^{6 / \alpha-1}\left(\gamma e^{-x^{2}}+\gamma^{2} e^{-2 x^{2}}+\cdots\right) d x\right]= \\
& =C(\alpha)\left(\frac{1}{\beta}\right)^{3 / \alpha} \sum_{n} \frac{\gamma^{n}}{n^{3 / \alpha}}=C(\alpha)\left(\frac{1}{\beta}\right)^{3 / \alpha} \zeta_{3 / \alpha}(\gamma) .
\end{aligned}
$$

Температура $T_{\mathrm{c}}$, при которой $\gamma=1$, называется критической температурой конденсации Бозе-Эйнштейна. Согласно выражению (20) для этой температуры $\rho=$ $C(\alpha)\left(k T_{\mathrm{c}}\right)^{3 / \alpha} \zeta_{3 / \alpha}(1)$. Отсюда

$$
T_{\mathrm{c}}=\frac{1}{k}\left(\frac{\rho}{C(\alpha) \zeta_{3 / \alpha}(1)}\right)^{\alpha / 3}
$$

Перепишем это выражение в виде

$$
T_{\mathrm{c}}=\left(\frac{\rho \alpha \Gamma(3 / 2)(2 \pi)^{3} E_{0}^{3 / \alpha} k_{0}^{-3}}{2 \pi^{3 / 2} \zeta_{3 / \alpha}(1)}\right)^{\alpha / 3}=\frac{E_{0}}{k_{\mathrm{B}}}\left(\frac{\rho \alpha \Gamma(3 / 2)(2 \pi)^{3} k_{0}^{-3}}{2 \pi^{3 / 2} \zeta_{3 / \alpha}(1)}\right)^{\alpha / 3} .
$$

Возьмем такую плотность, чтобы при $\alpha=2$ температура перехода была равна $3.2 \mathrm{~K}$ (критическая температура жидкого ${ }^{4} \mathrm{He}$ ). Эта плотность равна $\rho \approx 10^{26} \mathrm{M}^{-3}$. Ха- 


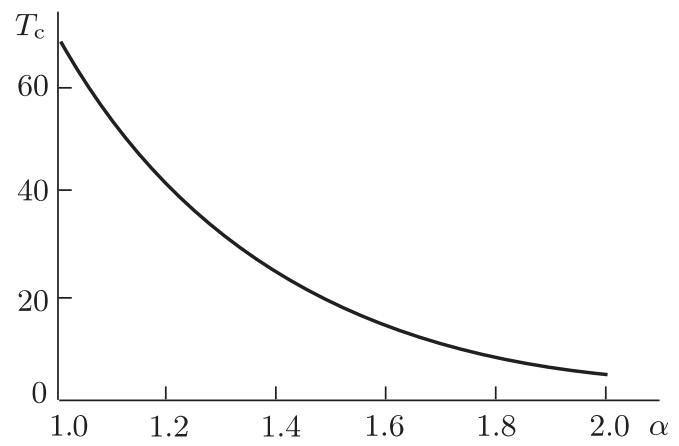

Рис. 4. Зависимость критической температуры от параметра $\alpha$.

рактеристическое волновое число выберем из следующих соображений. Для бозежидкости часто применяют аппроксимацию, в которой молекулы взаимодействуют как твердые сферы диаметром $b=2.56 \cdot 10^{-10}$ м. Соответственно для характеристического волнового числа имеем $k_{0}=2 \pi / b \approx 2.45 \cdot 10^{10} \mathrm{M}^{-1}$. Тогда, рассматривая газ атомов, массы которых порядка массы атомов ${ }^{4} \mathrm{He}\left(6.64 \cdot 10^{-27}\right.$ кг), и пользуясь формулой $E_{0}=\hbar^{2} k_{0}^{2} / 2 m$, находим характеристическую энергию $E_{0} \approx 0.121$ эВ. Пользуясь этими постоянными, можно построить зависимость критической температуры от параметра $\alpha$ (рис. 4).

Большой интерес вызывает то, что критическая температура конденсации оказывается довольно сильно повышенной при значениях $\alpha<2$. Этот вывод интересен для теории высокотемпературных сверхпроводников ввиду того, что электронный газ для сверхпроводников можно рассматривать как бозе-эйнштейновский конденсат куперовских пар, а критическую температуру конденсации - как температуру перехода в сверхпроводящее состояние.

\section{7. ЗАКЛЮЧЕНИЕ}

Из рассмотренного выше можно сделать вывод, что появление дробных операторов в уравнении для функции Грина связано с неидеальностью системы. Рассмотрение было проведено для общего случая. Оно может быть применено как к фермионному, так и к бозонному газам. Наиболее интересным, на наш взгляд, представляется применение дробно-дифференциального подхода к фононному газу. Как известно, неидеальность фононного газа, вызванная, в частности, сложностью структуры кристалла, приводит к ангармонизму, с которым связаны многие интересные эффекты. Теория ангармонизма дробно-степенно́го характера имеет свои особенности и может представлять большой интерес при описании реальных экспериментальных результатов. Так, элементарный расчет показывает, что дробно-дифференциальный подход дает широкий класс зависимостей теплоемкости от температуры. В то же время известно, что зависимость теплоемкости от температуры для сложных кристаллов не является однозначной функцией, а определяется типом кристалла и структурой. Последнее обстоятельство практически не описывается существующей теорией. Таким образом, дробно-дифференциальный подход в теории ангармонических эффектов в кристаллах представляет большой интерес. 


\section{Список литературы}

[1] И. М. Лифшиц, М. И. Каганов, УФН, 69:11 (1959), 419-458.

[2] Ф. Г. Басс, Е. А. Рубинштейн, ФТT, 19:5 (1977), 1379-1388.

[3] Ф. Г. Басс, В. В. Конотоп, А. П. Панчеха, ЖЭТФ, 96:5 (1989), 1869-1879.

[4] Ф. Г. Басс, А. П. Панчеха, ЖЭТФ, 99:6 (1991), 1711-1717.

[5] R. Hilfer (ed.), Applications of Fractional Calculus in Physics, World Scientific, Singapore, 2000.

[6] В. В. Учайкин, Метод дробных производных, изд-во “Артишок", Ульяновск, 2008.

[7] В. В. Учайкин, ЖЭТФ, 124:4 (2003), 903-920.

[8] А.А. Станиславский, ТМФ, 138:3 (2004), 491-507.

[9] Р. П. Мейланов, М. Р. Шабанова, ЖТФ, 81:7 (2011), 1-6.

[10] Р. Т. Сибатов, В. В. Учайкин, УФН, 179:10 (2009), 1079-1104.

[11] С.Ш. Рехвиашвили, ФТT, 49:4 (2007), 756-759.

[12] X.-B. Wang, J.-X. Li, Q. Jiang, Z.-H. Zhang, D.-C. Tian, Phys. Rev., 49:14 (1994), 9778-9781.

[13] A. V. Milovanov, J. J. Rasmussen, Phys. Rev. B, 66:13 (2002), 134505, 11 pp., arXiv: cond-mat/0201504.

[14] F. J. Dyson, Commun. Math. Phys., 12:2 (1969), 91-107.

[15] F. J. Dyson, Commun. Math. Phys., 12:3 (1969), 212-215.

[16] F. J. Dyson, Commun. Math. Phys., 21:4 (1971), 269-283.

[17] N. Laskin, G. Zaslavsky, Physica A, 368:1 (2006), 38-54, arXiv: nlin/0512010.

[18] В. С. Владимиров, И. В. Волович, Е. И. Зеленов, p-Адический анализ и математическая физика, Наука, М., 1994.

[19] N. Laskin, Phys. Lett. A, 268:4-6 (2000), 298-305, arXiv: hep-ph/9910419.

[20] N. Laskin, Phys. Rev. E, 62:3 (2000), 3135-3145, arXiv: 0811.1769.

[21] В. М. Елеонский, В. Г. Королев, Н. Е. Кулагин, Писъма в ЖЭТФ, 76:12 (2002), 859-862.

[22] V.E. Tarasov, Phys. Rev. E, 71:1 (2005), 011102, 12 pp., arXiv: cond-mat/0505720.

[23] V.E. Tarasov, Chaos, 16:3 (2006), 033108, 7 pp., arXiv: 0710.1807.

[24] V.E. Tarasov, Modern Phys. Lett. B, 21:5 (2007), 237-248, arXiv: 0711.0859.

[25] K. S. Novoselov, A. K. Geim, S. V. Morozov, D. Jiang, Y. Zhang, S. V. Dubonos, I. V. Grigorieva, A. A. Firsov, Science, 306:5696 (2004), 666-669, arXiv: cond-mat/0410550.

[26] K. S. Novoselov, A. K. Geim, S. V. Morozov, D. Jiang, M. I. Katsnelson, I. V. Grigorieva, S. V. Dubonos, A. A. Firsov, Nature, 438:7065 (2005), 197-200.

[27] A. H. Castro Neto, F. Guinea, N. M. R. Peres, K. S. Novoselov, A. K. Geim, Rev. Modern Phys., 81:1 (2009), 109-162, arXiv: 0709.1163.

[28] 3. З. Алисултанов, Р. П. Мейланов, ТМФ, 171:3 (2012), 404-416.

[29] С. Г. Самко, Ф.Ф. Килбас, О. И. Маричев, Интегралы и производные дробного порядка и некоторые приложения, Наука и техника, Минск, 1987.

[30] А. Б. Алхасов, Р. П. Мейланов, М. Р. Шабанова, ИФЖ, 84:2 (2011), 309-317.

[31] Л. Каданов, Г. Бейм, Квантовая статистическая механика. Методы функиий Грина в теории равновесных и неравновесных процессов, Мир, М., 1964.

[32] Р. Балеску, Статистическая механика заряженных частии, Мир, М., 1967.

[33] И.Г. Каплан, Введение в теорию межмолекулярных взаимодействий, Наука, М., 1982.

[34] W. Weibull, J. Appl. Mech., 18:3 (1951), 293-297. 